\title{
Article \\ General Summation Formulas Contiguous to the $q$-Kummer Summation Theorems and Their Applications
}

\author{
Yashoverdhan Vyas ${ }^{1}\left(\mathbb{D}\right.$, Hari M. Srivastava ${ }^{2,3,4,5, *(\mathbb{D})}$, Shivani Pathak ${ }^{1}\left(\mathbb{D}\right.$ and Kalpana Fatawat ${ }^{6}$ (D) \\ 1 Department of Mathematics, School of Engineering, Sir Padampat Singhania University, Bhatewar, \\ Udaipur 313601, Rajasthan, India; yashoverdhan.vyas@spsu.ac.in (Y.V.); shivani.bhatt@spsu.ac.in (S.P.) \\ 2 Department of Mathematics and Statistics, University of Victoria, Victoria, BC V8W 3R4, Canada \\ 3 Department of Medical Research, China Medical University, Taichung 40402, Taiwan \\ 4 Department of Mathematics and Informatics, Azerbaijan University, 71 Jeyhun Hajibeyli Street, \\ AZ1007 Baku, Azerbaijan \\ 5 Section of Mathematics, International Telematic University Uninettuno, I-00186 Rome, Italy \\ 6 Techno India NJR Institute of Technology, Plot SPL-T, Bhamashah (RIICO) Industrial Area, Kaladwas, \\ Udaipur 313003, Rajasthan, India; kalpana.fatawat@technonjr.org \\ * Correspondence: harimsri@math.uvic.ca
}

Citation: Vyas, Y.; Srivastava, H.M.;

Pathak, S.; Fatawat, K. General

Summation Formulas Contiguous to the $q$-Kummer Summation Theorems and Their Applications. Symmetry 2021, 13, 1102. https://doi.org/ 10.3390/sym13061102

Academic Editor: Alexei Kanel-Belov

Received: 28 May 2021

Accepted: 16 June 2021

Published: 21 June 2021

Publisher's Note: MDPI stays neutral with regard to jurisdictional claims in published maps and institutional affiliations.

Copyright: () 2021 by the authors Licensee MDPI, Basel, Switzerland. This article is an open access article distributed under the terms and conditions of the Creative Commons Attribution (CC BY) license (https:// creativecommons.org/licenses/by/ $4.0 /)$.

\begin{abstract}
This paper provides three classes of $q$-summation formulas in the form of general contiguous extensions of the first $q$-Kummer summation theorem. Their derivations are presented by using three methods, which are along the lines of the three types of well-known proofs of the $q$-Kummer summation theorem with a key role of the $q$-binomial theorem. In addition to the $q$-binomial theorem, the first proof makes use of Thomae's $q$-integral representation and the second proof needs Heine's transformation. Whereas the third proof utilizes only the $q$-binomial theorem. Subsequently, the applications of these summation formulas in obtaining the general contiguous extensions of the second and the third $q$-Kummer summation theorems are also presented. Furthermore, the investigated results are specialized to give many of the known as well as presumably new $q$-summation theorems, which are contiguous to the three $q$-Kummer summation theorems. This work is motivated by the observation that the basic (or $q$-) series and basic (or $q$-) polynomials, especially the basic (or $q$-) gamma and $q$-hypergeometric functions and basic (or $q$-) hypergeometric polynomials, are applicable particularly in several diverse areas including Number Theory, Theory of Partitions and Combinatorial Analysis as well as in the study of Combinatorial Generating Functions. Just as it is known in the theory of the Gauss, Kummer (or confluent), Clausen and the generalized hypergeometric functions, the parameters in the corresponding basic or quantum (or $q$-) hypergeometric functions are symmetric in the sense that they remain invariant when the order of the $p$ numerator parameters or when the order of the $q$ denominator parameters is arbitrarily changed. A case has therefore been made for the symmetry possessed not only by hypergeometric functions and basic or quantum (or $q$-) hypergeometric functions, which are studied in this paper, but also by the symmetric quantum calculus itself.
\end{abstract}

Keywords: symmetric quantum calculus; quantum or basic (or $q$-) hypergeometric series; $q$-Binomial theorem; $q$-Kummer summation theorem; Thomae's $q$-integral representation; Heine's transformation; $q$-Kummer second and third summation theorems

MSC: Primary 33D05; 33D15; Secondary 05A30; 11B65; 33C05; 33C20

\section{Introduction, Motivation and Preliminaries}

The celebrated Gauss hypergeometric function ${ }_{2} F_{1}$, the Kummer (or confluent) hypergeometric function ${ }_{1} F_{1}$, the Clausen hypergeometric function ${ }_{3} F_{2}$, and various other mathematical functions of hypergeometric type, are all contained in the generalized hypergeometric function ${ }_{r} F_{s}$, involving $r$ numerator parameters $a_{1}, \cdots, a_{r}$ and $s$ denominator 
parameters $b_{1}, \cdots, b_{s}$, as special cases. For definition and other details including the criteria to ensure the convergence of the series defining the generalized hypergeometric function ${ }_{r} F_{S}$, we refer the reader to [1-4]).

The generalized basic or quantum (or q-) hypergeometric series is defined by (for details, see $[2,3,5-7])$.

$$
\begin{gathered}
{ }_{r} \Phi_{s}\left[\begin{array}{c}
a_{1}, \cdots, a_{r} ; \\
b_{1}, \cdots, b_{s} ;
\end{array}\right] \\
\quad=\sum_{n=0}^{\infty} \frac{\left(a_{1} ; q\right)_{n} \cdots\left(a_{r} ; q\right)_{n}}{\left(b_{1} ; q\right)_{n} \cdots\left(b_{s} ; q\right)_{n}} \frac{z^{n}}{(q ; q)_{n}}\left[(-1)^{n} q^{\left(\begin{array}{c}
n \\
2
\end{array}\right)}\right]^{1+s-r},
\end{gathered}
$$

where the $q$-shifted factorials are given by

$$
(a ; q)_{0}:=1 \quad \text { and } \quad(a ; q)_{n}:=(1-a) \cdots\left(1-a q^{n-1}\right) \quad(n \in \mathbb{N}),
$$

where, and in what follows, the symbol $\mathbb{N}_{0}$ denotes the set of 0 -inclusive natural numbers and $\mathbb{N}=\mathbb{N}_{0} \backslash\{0\}$. A detailed discussion on the convergence of the series (1) can be found in [3] (p. 347), [6] (pp. 22-23) and [7] (pp. 4-5).

For the $q$-shifted factorials (2), we use a compact notation as follows:

$$
\left(a_{1}, \cdots, a_{r} ; q\right)_{n}:=\left(a_{1} ; q\right)_{n} \cdots\left(a_{r} ; q\right)_{n} \quad\left(n \in \mathbb{N}_{0}\right)
$$

In addition, when $n$ tends to infinity, we use the following notations:

$$
(a ; q)_{\infty}:=\prod_{n=0}^{\infty}\left(1-a q^{n}\right)
$$

and

$$
\left(a_{1}, \cdots, a_{r} ; q\right)_{\infty}:=\left(a_{1} ; q\right)_{\infty} \cdots\left(a_{r} ; q\right)_{\infty} .
$$

For further identities involving the $q$-shifted factorials, we refer the reader to [7] (pp. 351-352, Appendix I). Many of these identities for $q$-shifted factorials will be freely used in our presentation here.

This article is motivated essentially by the fact that the basic (or $q$-) series, basic (or $q$-) polynomials and basic (or $q$-) calculus, specifically the basic (or $q$-) hypergeometric functions and the basic (or $q$-) hypergeometric polynomials have demonstrated applications in the area of Number Theory such as, for example, the Theory of Partitions and are also found to be useful in a wide range of fields (see, for details, [3] (pp. 350-351), [8] (p. 328), and [9] (p. 1817); see also the references cited therein). Further motivation for studying such quantum (or $q$-) hypergeometric functions in this paper can be found in the book chapter, entitled "Symmetric quantum calculus" in [10].

In the theory and applications of the Gauss hypergeometric function ${ }_{2} F_{1}$, the Kummer (or confluent) hypergeometric function ${ }_{1} F_{1}$, the Clausen hypergeometric function ${ }_{3} F_{2}$, and various other mathematical functions of hypergeometric type, contained as special cases in the generalized hypergeometric function ${ }_{r} F_{S}$, it is often useful to know that the sets of the $r$ numerator parameters $a_{1}, \cdots, a_{r}$ and the $s$ denominator parameters $b_{1}, \cdots, b_{s}$, are symmetric individually. The same is true individually for the sets of the numerator parameters and the denominator parameters in the their quantum (or $q^{-}$) extensions involved in Equation (1), which we have considered in this paper.

There are many classical summation and transformation theorems for the hypergeometric functions as listed by Slater (see, for example, [2] (Appendix III, pp. 243-245) and [7] (pp. 354-356, Appendix II); see also [2] (p. 247, Appendix IV) and [5]).

The present work deals with the contiguous extensions of the basic (or $q$-) analogues of three hypergeometric summation theorems due to Kummer [11]. The three classical, 
well-known and celebrated hypergeometric summation theorems for the ${ }_{2} F_{1}(z)$ series with $z=-1$ and $z=\frac{1}{2}$ include Kummer's first summation theorem for $z=-1$ (see [2] (p. 243, Equation (III.5)) and [1] (p. 68, Theorem 26)) and the two summation theorems for $z=\frac{1}{2}$ [2] (p. 243, Equations (III.6) and (III.7)). All three of these hypergeometric summation theorems were given, in fact, by Kummer [11] (p. 134, Entries 1, 2 and 3) in 1836. We refer to these last three results as Kummer's first summation theorem, Kummer's second summation theorem and Kummer's third summation theorem, respectively (see also the remarks by Choi et al. [12] about incorrectly giving credit instead to Gauss and Bailey for the last two Kummer summation theorems for the ${ }_{2} F_{1}(z)$ series with $z=\frac{1}{2}$ ). Of these three known summation theorems, until 1973, only a $q$-analogue of Kummer's first summation theorem was known as follows (see Reference [13] (p. 711); see also [14] (p. 173)):

$$
{ }_{2} \Phi_{1}\left[\begin{array}{l}
a, b ; \\
\frac{a q}{b} ;
\end{array},-\frac{q}{b}\right]=\frac{(-q ; q)_{\infty}\left(a q, \frac{a q^{2}}{b^{2}} ; q^{2}\right)_{\infty}}{\left(\frac{a q}{b},-\frac{q}{b} ; q\right)_{\infty}} .
$$

The proof of the result stated in Equation (6) was based upon the specialization of parameters in Jackson's summation of the well-poised ${ }_{6} \Phi_{5}$ [7] (p. 356, Equation (II.20)). Later on, in two different papers, Andrews (see [15,16]) described two proofs for the $q$-analogue of Kummer's first summation theorem. Andrews [15] employed series rearrangement and the $q$-binomial theorem three times and, in [16], he used Heine's transformation and the $q$-binomial theorem, where the $q$-binomial theorem [7] (p. 8, Equation (1.3.2)) and the Heine's transformation [7] (p. 13, Equation (1.4.1)) are as follows:

$$
\sum_{n=0}^{\infty} \frac{(a ; q)_{n}}{(q ; q)_{n}} z^{n}=\frac{(a z ; q)_{\infty}}{(z ; q)_{\infty}} \quad(\max \{|z|,|q|\}<1)
$$

and

$$
{ }_{2} \Phi_{1}\left[\begin{array}{c}
a, b ; \\
c ; \\
c ;
\end{array}\right]=\frac{(b, a z ; q)_{\infty}}{(c, z ; q)_{\infty}}{ }_{2} \Phi_{1}\left[\begin{array}{c}
\frac{c}{b}, z ; \\
a z ;
\end{array}\right] .
$$

Andrews [15] also derived the $q$-analogue of Kummer's second summation theorem [6] (p. 31, Equation (1.4.6.5)):

$$
{ }_{2} \Phi_{2}\left[\begin{array}{c}
a, b ; \\
\sqrt{q a b},-\sqrt{q a b} ;
\end{array},-q\right]=\frac{(-q ; q)_{\infty}\left(a q, b q ; q^{2}\right)_{\infty}}{\left(q a b ; q^{2}\right)_{\infty}},
$$

and the $q$-analogue of Kummer's third summation theorem (see [6] (p. 31, Equation (1.4.7.2)) and [7] (p. 354, Equation (II.10))):

$$
{ }_{2} \Phi_{2}\left[\begin{array}{c}
a, \frac{q}{a} ; q,-b \\
b,-q ;
\end{array}\right]=\frac{\left(a b, \frac{q b}{a} ; q^{2}\right)_{\infty}}{(b ; q)_{\infty}}
$$

by utilizing the following (Jackson's) transformation [17]:

$$
{ }_{2} \Phi_{2}\left[\begin{array}{c}
a, \frac{c}{b} ; \\
c, a z ;
\end{array}\right]=\frac{(z ; q)_{\infty}}{(a z ; q)_{\infty}}{ }_{2} \Phi_{1}\left[\begin{array}{c}
a, b ; \\
c ;
\end{array}\right]
$$

and the $q$-Kummer first summation theorem (6). We refer to the results (9) and (10) as the $q$ Kummer second summation theorem and the $q$-Kummer third summation theorem, respectively. 
Contributions towards contiguous extensions of summation and transformation formulas for basic (or $q$-) generalized hypergeometric series appear to be a lot less extensive. For example, in the context of $q$-Kummer's theorems (6), (9) and (10), Kim et al. [18] re-derived the $q$-analogue of Kummer's first theorem (6) by using Thomae's $q$-integral representation given by Equation (13) and also obtained two particular contiguous results [18] (p. 154, Equations (3.1) and (3.2)). In the same context, recently, Harsh et al. [19] (pp. 81-92) derived some more particular contiguous extensions of the $q$-Kummer's first theorem (6), one-by-one from certain $q$-contiguous relations. In another paper, Harsh et al. [20] (pp. 29-31) derived some particular contiguous extensions of the $q$-Kummer second summation theorem (9) and the $q$-Kummer third summation theorem (10) by implementing the summation formulas contiguous to the $q$-Kummer first theorem, derived in the previous paper [19] (pp. 81-92), along the lines of the derivations by Andrews [15]. The particular contiguous extensions for the three $q$-Kummer summation theorems, investigated in $[19,20]$, are very limited in numbers and are in scattered forms due to the one-by-one use of $q$-contiguous relations. In conclusion, as per our observations, the general contiguous extensions for the three $q$-Kummer summation theorems, which are presented in this paper in the form of Theorems 1 to 6 have not appeared previously in the literature. For some recent developments on the basic (or $q$-) analogues of the known results on summations, transformations and reductions for ordinary hypergeometric series in one, two and more variables (see, especially for a variety of techniques used in the $q$-analysis [21-25]).

A thorough review of the existing literature shows that not all of the results in the theory of ordinary hypergeometric series have their basic (or $q$-) analogues. For example, in [26], certain new ordinary hypergeometric identities are derived and the investigation of their $q$-analogues has been placed as an open problem. In order to bridge this type of important gaps in the existing literature, several workers have attempted to find transformations connecting basic hypergeometric series with different bases (see [27]). Notably, the first attempt that has borne ample fruits is through a paper by Singh [28] in which he discovered a relation between two ${ }_{4} \Phi_{3}$ series (see [7] (p. 361, Equation (III.21))) with bases $q$ and $q^{2}$, respectively. This solitary transformation filled in a century-old gap in the theory of basic (or $q$-) series. In fact, it helped in proving an entire class of $q$-identities known as basic analogues of identities of the Cayley-Orr type, which owe their origin to certain problems in Stellar Physics. It is believed that the $q$-results, which we have derived in this paper, are potentially useful in some or the other areas listed above as well as in, for example, [3] (pp. 350-351) and [9] (p. 1817).

Our objective here is to investigate the compact and closed-form representations of the general contiguous extensions (with bases $q$ and $q^{2}$ ) of the $q$-Kummer first summation theorem (see Section 2, Theorems 1-3), and the $q$-Kummer second and third summation theorems (see Section 3, Theorems 4-6). In particular, Theorems 1 and 2 (see Section 2) of this paper provide two general contiguous extensions of the $q$-Kummer first summation theorem in the form of general summations for the following ${ }_{2} \Phi_{1}$ basic (or $q$-) hypergeometric series:

$$
{ }_{2} \Phi_{1}\left[\begin{array}{c}
a, b ; \\
\frac{a q^{1 \pm k}}{b} ; q,-\frac{q}{b}
\end{array}\right] \quad\left(k \in \mathbb{N}_{0}\right) .
$$

The limiting cases of Theorems 1 and 2 when $q \rightarrow 1$ produce the general contiguous extensions of Kummer's theorem due to Choi et al. [29] (pp. 1523-1524, Equations (2.2) and (2.3), respectively). Interestingly, Theorem 3 provides another $q$-analogue of the result due to Choi et al. [29] (p. 1524, Equation (2.3)) (see Section 2). Further, in Section 3, Theorems 1 to 3 are applied in conjunction with the Jackson's transformation (11) to provide the generalizations of the $q$-Kummer second and third summation theorems in the form of Theorems 4 to 6 . 


\section{General Contiguous Extensions of the $q$-Kummer First Theorem: Statements and Derivations}

In this section, we should first add a brief remark about the need for multiple proofs of a given result. In the literature in the mathematical sciences, one can find numerous such instances. Multiple proofs of a result provide different viewpoints as well as independent ways to tackle the problem and, therefore, encourage and motivate the use of these different ways to tackle other problems as well. For example, in the literature on generating functions, there are presumably as many as five independent proofs of Jacobi's celebrated generating function for the Jacobi polynomials, of which the fifth proof was given by Srivastava [30]. The methodology and techniques, which were used in these five independent proofs, have since been applied in deriving other families of generating functions as well. We also observe that the three techniques used in $[15,16,18]$, respectively, will be used to present the three proofs of the general contiguous extensions of the first $q$-Kummer summation Theorem (6) in Section 2.

- The first technique is based on the earlier work [18], which used Thomae's $q$-integral representation [7] (p. 24, Equation (1.11.9)), that is,

$$
{ }_{2} \Phi_{1}\left[\begin{array}{c}
a, b ; \\
c ;
\end{array}\right]=\frac{\Gamma_{q}(c)}{\Gamma_{q}(b) \Gamma_{q}(c-b)} \int_{0}^{1} t^{b-1} \frac{\left(t z q^{a}, t q ; q\right)_{\infty}}{\left(t z, t q^{c-b} ; q\right)_{\infty}} d_{q} t
$$

along with the application of the following definition of the $q$-gamma function $\Gamma_{q}(z)$ [7] (p. 20, Equation (1.10.1)) and Jackson's q-integral [7] (p. 23, Equation (1.11.1)):

$$
\Gamma_{q}(x)=\frac{(q ; q)_{\infty}}{\left(q^{x} ; q\right)_{\infty}}(1-q)^{1-x} \quad(0<q<1)
$$

and

$$
\int_{0}^{1} f(t) d_{q} t=(1-q) \sum_{n=0}^{\infty} f\left(q^{n}\right) q^{n}
$$

respectively. The derivation, which is discussed in Section 2 by using (13) (see First proof of Theorem 1), is comparatively more compact and brief than the proof given by Kim et al. [18];

- The second technique is based upon the earlier work [16], which used Heine's transformation (8) (see Second proof of Theorem 1);

- $\quad$ The third technique is based on the earlier work [15] which only used a three-times application of the $q$-binomial theorem (7) (see Third proof of Theorem 1).

All three proofs of the general contiguous extensions of the first $q$-Kummer summation theorem (6), which we will present in this section, require one additional use of the $q$-binomial theorem (7) compared to the corresponding proofs of $q$-Kummer first summation theorem (6), presented in earlier works $[15,16,18]$. In comparison with some of the other methods leading to basic (or $q$-) extensions of hypergeometric summation and transformation fomulas, the above-mentioned methodology and techniques, which we use in this paper, are demonstrably much more widely applicable and in more general situations as well.

We now state and prove the main results of this paper in the form of Theorems 1-3 along with their derivations. The $q$-binomial theorem (7) plays a key role in all the derivations presented here. However, in addition, the first proof uses Thomae's $q$-integral and the second proof needs Heine's transformation, whereas the third proof depends only on the $q$-binomial theorem (7).

Theorem 1. For any $k \in \mathbb{N}_{0}$, the following assertion holds true: 


$$
\begin{gathered}
{ }_{2} \Phi_{1}\left[\begin{array}{c}
a, b ; \\
\frac{a q^{1+k}}{b} ; q,-\frac{q}{b}
\end{array}\right]=\frac{\left(a, \frac{q^{1+k}}{b},-q ; q\right)_{\infty}}{\left(\frac{a q^{1+k}}{b} ; q\right)_{\infty}\left(\frac{q^{2}}{b^{2}} ; q^{2}\right)_{\infty}} \\
\cdot \sum_{j=0}^{k} \frac{\left(q^{-k} ; q\right)_{j}}{(q ; q)_{j}}\left(\frac{q^{1+k}}{b}\right)^{j} \frac{\left(\frac{a q^{j+2}}{b^{2}} ; q^{2}\right)_{\infty}}{\left(a q^{j} ; q^{2}\right)_{\infty}}
\end{gathered}
$$

provided that $\left|\frac{q}{b}\right|<1$.

First Proof of Theorem 1.

Representing the left-hand side of Theorem 1 by $\Omega$ and using the Thomae's $q$-integral (13), we obtain:

$$
\Omega=\frac{\Gamma_{q}\left(\frac{a q^{1+k}}{b}\right)}{\Gamma_{q}(a) \Gamma_{q}\left(\frac{q^{1+k}}{b}\right)} \int_{0}^{1} t^{a-1} \frac{(t q,-t q ; q)_{\infty}}{\left(-t q^{1-b}, t q^{1+k-b} ; q\right)_{\infty}} d_{q} t
$$

Applying an elementary identity for $q$-shifted factorials to the integrand of the previous result (Equation (17)) leads to

$$
\Omega=\frac{\Gamma_{q}\left(\frac{a q^{1+k}}{b}\right)}{\Gamma_{q}(a) \Gamma_{q}\left(\frac{q^{1+k}}{b}\right)} \int_{0}^{1} t^{a-1} \frac{\left(t^{2} q^{2} ; q^{2}\right)_{\infty}}{\left(\frac{t^{2} q^{2}}{b^{2}} ; q^{2}\right)_{\infty}} \frac{\left(t q^{1-b} ; q\right)_{\infty}}{\left(t q^{1+k-b} ; q\right)_{\infty}} d_{q} t .
$$

Next, by applying the definitions of the q-gamma function (14) and Jackson's $q$ integral (15) to the right-hand side of Equation (18), we obtain

$$
\Omega=\frac{\left(a, \frac{q^{1+k}}{b} ; q\right)_{\infty}}{\left(\frac{a q^{1+k}}{b}, q ; q\right)_{\infty}} \sum_{n=0}^{\infty} \frac{\left(q^{2 n+2} ; q^{2}\right)_{\infty} q^{a n}}{\left(\frac{q^{2 n+2}}{b^{2}} ; q^{2}\right)_{\infty}} \frac{\left(\frac{q^{1+n}}{b} ; q\right)_{\infty}}{\left(\frac{q^{1+k+n}}{b} ; q\right)_{\infty}} .
$$

Now, the application of the $q$-binomial theorem (7) to the last fraction of right-hand side of Equation (19), yields:

$$
\Omega=\frac{\left(a, \frac{q^{1+k}}{b} ; q\right)_{\infty}}{\left(\frac{a q^{1+k}}{b}, q ; q\right)_{\infty}} \sum_{j=0}^{k} \frac{\left(q^{-k} ; q\right)_{j}}{(q ; q)_{j}}\left(\frac{q^{1+k}}{b}\right)^{j} \sum_{n=0}^{\infty} \frac{\left(q^{2 n+2} ; q^{2}\right)_{\infty} q^{(a+j) n}}{\left(\frac{q^{2 n+2}}{b^{2}} ; q^{2}\right)_{\infty}} .
$$

Finally, another application of the $q$-binomial theorem (7), this time for the summation over $n$, leads to Theorem 1. 
Second Proof of Theorem 1.

Interchanging $a$ and $b$, in the Heine's transformation (8), and letting

$$
c=\frac{a q^{1+k}}{b} \quad \text { and } \quad z=-\frac{q}{b}
$$

and then denoting the resulting left-hand side by $\Omega$, we obtain

$$
\Omega=\frac{(a,-q ; q)_{\infty}}{\left(\frac{a q^{1+k}}{b},-\frac{q}{b} ; q\right)_{\infty}} \sum_{n=0}^{\infty} \frac{\left(\frac{q^{1+k}}{b},-\frac{q}{b} ; q\right)_{n} q^{a n}}{\left(q^{2} ; q^{2}\right)_{n}} .
$$

Next, by using known identities involving $q$-shifted factorials and the $q$-binomial theorem (7), to the right-hand side of Equation (21), we find that

$$
\begin{aligned}
\Omega= & \frac{\left(a,-q, \frac{q^{1+k}}{b} ; q\right)_{\infty}}{\left(\frac{a q^{1+k}}{b} ; q\right)_{\infty}\left(\frac{q^{2}}{b^{2}} ; q^{2}\right)_{\infty}} \\
& \cdot \sum_{j=0}^{k} \frac{\left(q^{-k} ; q\right)_{j}}{(q ; q)_{j}}\left(\frac{q^{1+k}}{b}\right)^{j} \cdot \sum_{n=0}^{\infty} \frac{\left(\frac{q^{2}}{b^{2}} ; q^{2}\right)_{n}\left(q^{a+j}\right)^{n}}{\left(q^{2} ; q^{2}\right)_{n}},
\end{aligned}
$$

Finally, by another application of the $q$-binomial theorem (7), this time to the last term of the right-hand side of Equation (22), involving the summation over $n$, we are led easily to Theorem 1.

\section{Third Proof of Theorem 1.}

If we represent the left-hand side ${ }_{2} \Phi_{1}$ of Theorem 1 by $\Omega$, then, from the series form of ${ }_{2} \Phi_{1}$, we can write that:

$$
\Omega={ }_{2} \Phi_{1}\left[\begin{array}{c}
a, b ; \\
\frac{a q^{1+k}}{b} ;
\end{array} ;-\frac{q}{b}\right]=\sum_{n=0}^{\infty} \frac{(a ; q)_{n}(b ; q)_{n}\left(-\frac{q}{b}\right)^{n}}{\left(\frac{a q^{1+k}}{b} ; q\right)_{n}(q ; q)_{n}}
$$

Now, by first using a known identity for $q$-shifted factorials in

$$
(a ; q)_{n} \quad \text { and } \quad\left(\frac{a q^{1+k}}{b} ; q\right)_{n}
$$

and then applying the $q$-binomial theorem (7), we obtain 


$$
\begin{aligned}
\Omega= & \frac{(a ; q)_{\infty}}{\left(\frac{a q^{1+k}}{b} ; q\right)_{\infty}} \sum_{m=0}^{\infty} \frac{\left(\frac{q^{1+k}}{b} ; q\right)_{m}\left(a q^{n}\right)^{m}}{(q ; q)_{m}} \\
& \cdot \sum_{n=0}^{\infty} \frac{(b ; q)_{n}\left(-\frac{q}{b}\right)^{n}}{(q ; q)_{n}} .
\end{aligned}
$$

Next, a second application of the $q$-binomial theorem (7), this time to the last term of Equation (24), involving summation over $n$, yields

$$
\Omega=\frac{(a,-q ; q)_{\infty}}{\left(\frac{a q^{1+k}}{b},-\frac{q}{b} ; q\right)_{\infty}} \sum_{m=0}^{\infty} \frac{\left(\frac{q^{1+k}}{b} ; q\right)_{m}(a)^{m}}{(q ; q)_{m}} \frac{\left(-\frac{q}{b} ; q\right)_{m}}{(-q ; q)_{m}} .
$$

Now, if we apply the above-mentioned identity for $q$-shifted factorials to the terms of the last quotient of Equation (25), we obtain

$$
\Omega=\frac{(a,-q ; q)_{\infty}}{\left(\frac{a q^{1+k}}{b},-\frac{q}{b} ; q\right)_{\infty}} \sum_{m=0}^{\infty} \frac{\left(\frac{q^{2}}{b^{2}} ; q^{2}\right)_{m}(a)^{m}}{\left(q^{2} ; q^{2}\right)_{m}} \frac{\left(\frac{q^{1+k}}{b} ; q\right)_{m}}{\left(\frac{q}{b} ; q\right)_{m}} .
$$

Next, by applying a known identity involving $q$-shifted factorials and performing the third application of the $q$-binomial theorem (7), this time, to the last fraction of the right-hand side of Equation (26), yields

$$
\begin{aligned}
\Omega= & \frac{(a,-q ; q)_{\infty}}{\left(\frac{a q^{1+k}}{b},-\frac{q}{b} ; q\right)_{\infty}} \sum_{j=0}^{k} \frac{\left(q^{-k} ; q\right)_{j}}{(q ; q)_{j}\left(\frac{q}{b} ; q\right)_{k}}\left(\frac{q^{1+k}}{b}\right)^{j} \\
& \cdot \sum_{m=0}^{\infty} \frac{\left(\frac{q^{2}}{b^{2}} ; q^{2}\right)_{m} q^{(a+j) m}}{\left(q^{2} ; q^{2}\right)_{m}} .
\end{aligned}
$$

Finally, the fourth application of the $q$-binomial theorem (7), this time to the last term of Equation (27), involving summation over $m$, leads us to Theorem 1.

Remark 1. The case $k=0$ of Theorem 1 leads to the classical $q$-Kummer first summation theorem (6). The limiting case $q \rightarrow 1$ of Theorem 1 yields a result due to [29] ( $p$.1523, Equation (2.2)). 
Theorem 2. For any $k \in \mathbb{N}_{0}$, the following assertion holds true:

$$
\begin{aligned}
{ }_{2} \Phi_{1}\left[\begin{array}{c}
a, b ; \\
\frac{a q^{1-k}}{b} ;
\end{array} ;-\frac{q}{b}\right]=\frac{(a,-q ; q)_{\infty}}{\left(\frac{a q^{1-k}}{b},-\frac{q^{1-k}}{b} ; q\right)_{\infty}} \\
\quad \cdot \sum_{j=0}^{k} \frac{\left(q^{-k} ; q\right)_{j}}{(q ; q)_{j}}\left(-\frac{q}{b}\right)^{j} \frac{\left(\frac{a q^{j+2(1-k)}}{b^{2}} ; q^{2}\right)_{\infty}}{\left(a q^{j} ; q^{2}\right)_{\infty}},
\end{aligned}
$$

provided that $\left|\frac{q}{b}\right|<1$.

Theorem 3. For any $k \in \mathbb{N}_{0}$, the following assertion holds true:

$$
\begin{array}{r}
\left.{ }_{2} \Phi_{1}\left[\begin{array}{c}
a, b q^{-k} ; \\
\frac{a q}{b} ;
\end{array}\right]-\frac{q}{b}\right]=\frac{(a,-q ; q)_{\infty}}{\left(\frac{a q}{b},-\frac{q}{b} ; q\right)_{\infty}} \\
\cdot \sum_{j=0}^{k} \frac{\left(q^{-k} ; q\right)_{j}}{(q ; q)_{j}}(-q)^{j} \frac{\left(\frac{a q^{j+2}}{b^{2}} ; q^{2}\right)_{\infty}}{\left(a q^{j} ; q^{2}\right)_{\infty}},
\end{array}
$$

provided that $\left|\frac{q}{b}\right|<1$.

Proofs of Theorems 2 and 3.

Theorems 2 and 3 can also be proved along the lines of each of the above-mentioned three proofs of Theorem 1 . We choose to omit the analogous details which are involved in each of these three demonstrations.

Remark 2. The case $k=0$ of Theorem 2 leads to the classical $q$-Kummer first theorem (6). The limiting case of Theorem 2, when $q \rightarrow 1$, yields a known result [29] ( $p$. 1524, Equation (2.3)). The case $k=0$ of Theorem 3 leads to the classical $q$-Kummer first theorem (6). The limiting case of Theorem 3, when $q \rightarrow 1$, leads to a known result in [29] ( $p$. 1524, Equation (2.3)).

\section{General Contiguous Extensions of the $q$-Kummer Second Summation Theorem and the $q$-Kummer Third Summation Theorem}

This section presents the application of the general contiguous extensions of the $q$-Kummer first summation theorem.

Theorem 4. For any $k \in \mathbb{N}_{0}$, the following assertion holds true:

$$
\begin{aligned}
& { }_{2} \Phi_{2}\left[\begin{array}{c}
a, b ; \\
\sqrt{a b q^{1-k}},-\sqrt{a b q^{1-k}} ; q^{1-k}
\end{array}\right] \\
& =\frac{(a,-q ; q)_{\infty}}{\left(a b q^{1-k} ; q^{2}\right)_{\infty}} \sum_{j=0}^{k} \frac{\left(q^{-k} ; q\right)_{j}(-q)^{j}}{(q ; q)_{j}} \frac{\left(b q^{1+j-k} ; q^{2}\right)_{\infty}}{\left(a q^{j} ; q^{2}\right)_{\infty}} .
\end{aligned}
$$


Proof. Replacing $b$ by $\frac{c}{b}$ and then letting

$$
c=\sqrt{a b q^{1-k}} \quad \text { and } \quad z=-\sqrt{\frac{b q^{1-k}}{a}}
$$

in Jackson's transformation (11), we obtain

$$
\begin{aligned}
& { }_{2} \Phi_{2}\left[\begin{array}{c}
a, b ; \\
\sqrt{a b q^{1-k}},-\sqrt{a b q^{1-k}} ;
\end{array}\right.
\end{aligned}
$$

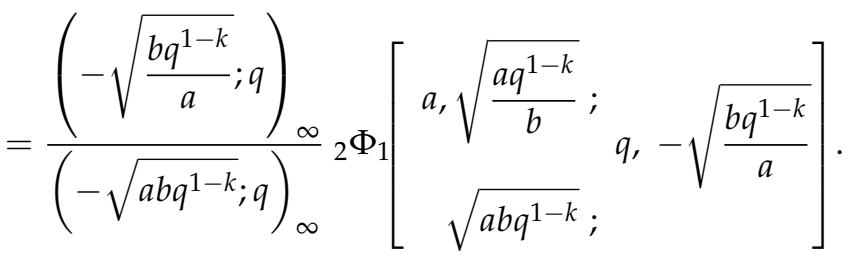

Finally, by applying a result, which is obtained by a replacement of $b$ by $\sqrt{\frac{a q^{1+k}}{b}}$ in Theorem 3, to the right-hand side of Equation (31), leads us to Theorem 4.

Remark 3. The case $k=0$ of Theorem 4 leads us to the q-Kummer second summation theorem (9). The limiting case of Theorem 4 , when $q \rightarrow 1$, gives an additional result in the form of a general contiguous extension of Kummer's second summation theorem.

Theorem 5. For any $k \in \mathbb{N}_{0}$, the following assertion holds true:

$$
\begin{aligned}
&{ }_{2} \Phi_{2}\left[\begin{array}{c}
\left.a, \frac{q^{1+k}}{a} ; q,-\frac{b}{q^{k}}\right] \\
b,-q ;
\end{array}\right] \frac{\left(\frac{q^{1+k}}{a}, \frac{a b}{q^{1+k}} ; q\right)_{\infty}}{\left(b, \frac{q}{a} ; q\right)_{\infty}} \\
& \cdot \sum_{j=0}^{k} \frac{\left(q^{-k} ; q\right)_{j}\left(\frac{q^{1+k}}{a}\right)^{j}}{(q ; q)_{j}} \frac{\left(\frac{b q^{1+j-k}}{a} ; q^{2}\right)_{\infty}}{\left(a b q^{j-k-1} ; q^{2}\right)_{\infty}} .
\end{aligned}
$$

Proof. The choice $c=\frac{b q^{1+k}}{a}$ in Jackson's transformation (11), followed by a replacement of $b$ by $\frac{a b}{q^{1+k}}$ and the substitution $z=-\frac{q}{a}$, we obtain

$$
{ }_{2} \Phi_{2}\left[\begin{array}{c}
a, \frac{q^{1+k}}{a} ; q,-\frac{b}{q^{k}} \\
b,-q ;
\end{array}\right]=\frac{\left(-\frac{q}{a} ; q\right)_{\infty}}{(-q ; q)_{\infty}}{ }_{2} \Phi_{1}\left[\begin{array}{c}
a, \frac{a b}{q^{1+k}} ; q,-\frac{q}{a} \\
b ;
\end{array}\right]
$$

Finally, applying a result, which is obtained by an interchange of $a$ and $b$, followed by a replacement of $b$ by $\frac{a b}{q^{1+k}}$ in Theorem 1, to the right-hand side of Equation (33) leads to Theorem 5.

Remark 4. The case $k=0$ of Theorem 5 leads us to the $q$-Kummer third summation theorem (10). 
Remark 5. The limiting case $q \rightarrow 1$ of Theorem 5 gives an additional result in the form of a general contiguous extension of Kummer's third summation theorem.

Theorem 6. For any $k \in \mathbb{N}_{0}$, the following assertion holds true:

$$
\begin{aligned}
{ }_{2} \Phi_{2}\left[\begin{array}{c}
a, \frac{q^{1-k}}{a} ; q,-b q^{k} \\
b,-q ;
\end{array}\right]=\frac{\left(-\frac{q}{a}, \frac{a b}{q^{1-k}} ; q\right)_{\infty}}{\left(b,-\frac{q^{1-k}}{a} ; q\right)_{\infty}} \\
\\
\quad \cdot \sum_{j=0}^{k} \frac{\left(q^{-k} ; q\right)_{j}\left(-\frac{q}{a}\right)^{j}}{(q ; q)_{j}} \frac{\left(\frac{b q^{1+j-k}}{a} ; q^{2}\right)_{\infty}}{\left(a b q^{j+k-1} ; q^{2}\right)_{\infty}} .
\end{aligned}
$$

Proof. First of all, taking $c=\frac{b q^{1-k}}{a}$ in Jackson's transformation (11), followed by a replacement of $b$ by $\frac{a b}{q^{1-k}}$ and the substitution $z=-\frac{q}{a}$, we obtain a transformation from ${ }_{2} \Phi_{2}$ to ${ }_{2} \Phi_{1}$ similar to Equation (33). At the end, the result developed from Theorem 2, by interchanging $a$ and $b$ and then setting $b \mapsto \frac{a b}{q^{1-k}}$, can be applied to the right-hand side of the obtained transformation to yield Theorem 6 .

Remark 6. The case $k=0$ of Theorem 6 leads us to the $q$-Kummer third summation theorem (10). The limiting case of Theorem 6 , when $q \rightarrow 1$, gives an additional result in the form of another general contiguous extension of Kummer's third summation theorem.

\section{Special Cases and Additional $q$-Summations}

Several known as well as additional $q$-summations are presented in this section as special cases of Theorems 1 to 6 .

1. Theorem 1, for $k=1,2,3,4$ and 5, produces the results given in [19] (pp. 892-83, Equations (6.100) to (6.104)), respectively. For example, the case $k=1$ of Theorem 1 is as shown below [19] (p. 82, Equation (6.100)):

$$
\begin{aligned}
& { }_{2} \Phi_{1}\left[\begin{array}{l}
a, b ; \\
\frac{a q^{2}}{b} ;
\end{array} ;,-\frac{q}{b}\right]=\frac{(-q ; q)_{\infty}}{\left(1-\frac{q}{b}\right)\left(\frac{a q^{2}}{b},-\frac{q}{b} ; q\right)_{\infty}} \\
& \cdot\left[\left(a q, \frac{a q^{2}}{b^{2}} ; q^{2}\right)_{\infty}-\frac{q}{b}\left(a, \frac{a q^{3}}{b^{2}} ; q^{2}\right)_{\infty}\right] .
\end{aligned}
$$

In addition, for $k=6,7,8, \cdots$, Theorem 1 gives a number of additional $q$ summations. For instance, taking $k=6$ in Theorem 1 , yields

$$
\begin{aligned}
& { }_{2} \Phi_{1}\left[\begin{array}{l}
a, b ; \\
\frac{a q^{7}}{b} ;
\end{array} ;,-\frac{q}{b}\right]=\frac{\left(a, \frac{q^{7}}{b},-q ; q\right)_{\infty}}{\left(\frac{a q^{7}}{b} ; q\right)_{\infty}\left(\frac{q^{2}}{b^{2}} ; q^{2}\right)_{\infty}} \\
& \cdot \sum_{j=0}^{6} \frac{\left(q^{-6} ; q\right)_{j}}{(q ; q)_{j}}\left(\frac{q^{7}}{b}\right)^{j} \frac{\left(\frac{a q^{j+2}}{b^{2}} ; q^{2}\right)_{\infty}}{\left(a q^{j} ; q^{2}\right)_{\infty}} .
\end{aligned}
$$


Next, by splitting the summation over $j$ in two parts by selecting the even and odd values of $j$ and then applying the identities (4) and (2), followed by further simplifications, leads to the following presumably new result:

$$
\begin{aligned}
{ }_{2} \Phi_{1}\left[\begin{array}{cr}
a, b ; & q,-\frac{q}{b} \\
\frac{a q^{7}}{b} ;
\end{array}\right] \\
=\frac{1}{\left(1-\frac{q}{b}\right)\left(1-\frac{q^{2}}{b}\right)\left(1-\frac{q^{3}}{b}\right)\left(1-\frac{q^{4}}{b}\right)\left(1-\frac{q^{5}}{b}\right)\left(1-\frac{q^{6}}{b}\right)} \\
\cdot \frac{(-q ; q)_{\infty}}{\left(\frac{a q^{7}}{b},-\frac{q}{b} ; q\right)_{\infty}}\left[\left(a q, \frac{a q^{8}}{b^{2}} ; q^{2}\right)_{\infty} X_{1}-\left(a, \frac{a q^{7}}{b^{2}} ; q^{2}\right)_{\infty} Y_{1}\right],
\end{aligned}
$$

where $X_{1}$ and $Y_{1}$ are as given below:

$$
\begin{gathered}
X_{1}=\left(1-\frac{a q^{6}}{b^{2}}\right)\left[\left(1-\frac{a q^{2}}{b^{2}}\right)\left(1-\frac{a q^{4}}{b^{2}}\right)+\lambda_{1}\left\{\left(1-\frac{a q^{4}}{b^{2}}\right)\right.\right. \\
\left.\left.+\frac{q^{7}\left(1-a q^{2}\right)}{b^{2}}\right\}\right]+\frac{q^{21}(1-a)\left(1-a q^{2}\right)\left(1-a q^{4}\right)}{b^{6}}
\end{gathered}
$$

and

$$
\begin{aligned}
Y_{1} & =\lambda_{1}^{\prime}\left\{\left(1-\frac{a q^{3}}{b^{2}}\right)\left(1-\frac{a q^{5}}{b^{2}}\right)+\frac{q^{14}(1-a q)\left(1-a q^{3}\right)}{b^{4}}\right\} \\
& +\frac{\left(1+q^{2}\right)\left(1+q^{3}\right)\left(1+q+q^{2}+q^{3}+q^{4}\right)(1-a q) q^{6}}{b^{3}}\left(1-\frac{a q^{5}}{b^{2}}\right)
\end{aligned}
$$

with

$$
\lambda_{1}=\frac{q^{3}\left(1+q^{2}+q^{4}\right)\left(1+q+q^{2}+q^{3}+q^{4}\right)(1-a)}{b^{2}}
$$

and

$$
\lambda_{1}^{\prime}=\frac{q\left(1+q+q^{2}+q^{3}+q^{4}+q^{5}\right)}{b} .
$$

2. Theorem 2, for $k=1,2,3,4$ and 5, produces the results given by [19] (pp. 83-84, Equations (6.105) to (6.109)), respectively, with Equations (6.106)-(6.109) in their corrected forms. In addition, for $k=6,7,8, \cdots$, Theorem 2 gives a number of additional $q$-summation formulas. For instance, the case when $k=6$ is given below:

$$
\begin{gathered}
{ }_{2} \Phi_{1}\left[\begin{array}{l}
a, b ; \\
\frac{a}{b q^{5}} ;
\end{array} ;,-\frac{q}{b}\right]=\frac{(-q ; q)_{\infty}}{\left(\frac{a}{b q^{5}},-\frac{1}{b q^{5}} ; q\right)_{\infty}} \\
\cdot\left[\left(a q, \frac{a}{b^{2} q^{4}} ; q^{2}\right)_{\infty} X_{2}+\left(a, \frac{a}{b^{2} q^{5}} ; q^{2}\right)_{\infty} Y_{2}\right],
\end{gathered}
$$

where $X_{2}$ and $Y_{2}$ are as given below:

$$
\begin{aligned}
X_{2} & =\left(1-\frac{a}{b^{2} q^{6}}\right)\left[\left(1-\frac{a}{b^{2} q^{10}}\right)\left(1-\frac{a}{b^{2} q^{8}}\right)+\lambda_{2}\left\{\left(1-\frac{a}{b^{2} q^{8}}\right)\right.\right. \\
& \left.\left.+\frac{\left(1-a q^{2}\right)}{b^{2} q^{5}}\right\}\right]+\frac{(1-a)\left(1-a q^{2}\right)\left(1-a q^{4}\right)}{b^{6} q^{15}}
\end{aligned}
$$


and

$$
\begin{aligned}
Y_{2} & =\lambda_{2}^{\prime}\left\{\left(1-\frac{a}{b^{2} q^{7}}\right)\left(1-\frac{a}{b^{2} q^{9}}\right)+\frac{(1-a q)\left(1-a q^{3}\right)}{b^{4} q^{10}}\right\} \\
& +\frac{\left(1+q^{2}\right)\left(1+q^{3}\right)\left(1+q+q^{2}+q^{3}+q^{4}\right)(1-a q)}{b^{3} q^{12}}\left(1-\frac{a}{b^{2} q^{7}}\right),
\end{aligned}
$$

with

$$
\lambda_{2}=\frac{\left(1+q^{2}+q^{4}\right)\left(1+q+q^{2}+q^{3}+q^{4}\right)(1-a)}{b^{2} q^{9}}
$$

and

$$
\lambda_{2}^{\prime}=\frac{\left(1+q+q^{2}+q^{3}+q^{4}+q^{5}\right)}{b q^{5}} .
$$

3. Theorem 3, for $k=1,2,3$ and 4, produces the results given by [19] (pp. 85-86, Equations (6.114) to (6.117)), respectively. In addition, for $k=5,6,7, \ldots$, Theorem 3 gives a number of additional $q$-summations. For instance, the case when $k=5$ yields

$$
\begin{aligned}
{ }_{2} \Phi_{1}\left[\begin{array}{c}
a, \frac{b}{q^{5}} ; \\
\\
\frac{a q}{b} ;
\end{array}\right]=\frac{(-q ; q)_{\infty}}{b} \\
\cdot\left[\left(a q, \frac{a q^{6}}{b^{2}} ; q^{2}\right)_{\infty} X_{3}+\left(a, \frac{a q^{7}}{b^{2}} ; q^{2}\right)_{\infty} Y_{3}\right],
\end{aligned}
$$

where $X_{3}$ and $Y_{3}$ are given by

$$
X_{3}=\left(1-\frac{a q^{2}}{b^{2}}\right)\left(1-\frac{a q^{4}}{b^{2}}\right)+\lambda_{3}\left\{\left(1+q^{2}\right)\left(1-\frac{a q^{4}}{b^{2}}\right)+\frac{\left(1-a q^{2}\right)}{q^{3}}\right\}
$$

and

$$
Y_{3}=\lambda_{3}^{\prime}\left\{\left(1-\frac{a q^{3}}{b^{2}}\right)+\frac{\left(1+q^{2}\right)(1-a q)}{q^{5}}\right\}+\frac{(1-a q)\left(1-a q^{3}\right)}{q^{10}},
$$

with

$$
\lambda_{3}=\frac{\left(1+q+q^{2}+q^{3}+q^{4}\right)(1-a)}{q^{7}}
$$

and

$$
\lambda_{3}^{\prime}=\frac{\left(1+q+q^{2}+q^{3}+q^{4}\right)}{q^{4}}\left(1-\frac{a q^{5}}{b^{2}}\right) .
$$

4. Theorem 4, for $k=1,2$ and 3, produces the results given by [20] (pp. 29-30, Equations (23)-(25)), respectively. In addition, for $k=4,5,6, \cdots$, Theorem 4 gives a number of additional $q$-summations. For instance, the case when $k=4$ yields

$$
\begin{gathered}
{ }_{2} \Phi_{2}\left[\begin{array}{c}
a, b ; \\
\sqrt{\frac{a b}{q^{3}}},-\sqrt{\frac{a b}{q^{3}}} ; q,-\frac{1}{q^{3}}
\end{array}\right]=\frac{(-q ; q)_{\infty}}{\left(\frac{a b}{q^{3}} ; q\right)_{\infty}} \\
\cdot\left[\left(a q, b q ; q^{2}\right)_{\infty} X_{4}+\left(a, b ; q^{2}\right)_{\infty} Y_{4}\right],
\end{gathered}
$$

where $X_{4}$ and $Y_{4}$ are as given below:

$$
X_{4}=\left(1-\frac{b}{q}\right) \lambda_{4}+\frac{(1-a)}{q^{3}} \lambda_{4}^{\prime}
$$

and

$$
Y_{4}=\frac{(1+q)\left(1+q^{2}\right)}{q^{3}}\left\{\left(1-\frac{b}{q^{2}}\right)+\frac{(1-a q)}{q^{3}}\right\}
$$


with

$$
\lambda_{4}=\left\{\left(1-\frac{b}{q^{3}}\right)+\frac{(1-a)}{q}\right\}
$$

and

$$
\lambda_{4}^{\prime}=\left\{\left(1-\frac{b}{q}\right) \frac{\left(1+q+2 q^{2}+q^{3}\right)}{q^{2}}+\frac{\left(1-a q^{2}\right)}{q^{3}}\right\} .
$$

5. Theorem 5, for $k=1$ and 2, produces the results given in [20] (p. 30, Equations (26) and (27)), respectively. In addition, for $k=3,4,5, \cdots$, Theorem 5 gives a number of additional $q$-summations. For instance, the case when $k=3$ yields

$$
\begin{gathered}
{ }_{2} \Phi_{2}\left[\begin{array}{c}
a, \frac{q^{4}}{a} ; q,-\frac{b}{q^{3}} \\
b,-q ;
\end{array}\right]=\frac{1}{\left(1-\frac{q}{a}\right)\left(1-\frac{q^{2}}{a}\right)\left(1-\frac{q^{3}}{a}\right)(b ; q)_{\infty}} \\
\cdot\left[\left(\frac{b}{a}, \frac{a b}{q^{3}} ; q^{2}\right)_{\infty} X_{5}-\left(\frac{b q}{a}, \frac{a b}{q^{4}} ; q^{2}\right)_{\infty} Y_{5}\right],
\end{gathered}
$$

where $X_{5}$ and $Y_{5}$ are given by

$$
X_{5}=\left(1-\frac{b}{a q^{2}}\right)+\frac{\left(1+q+q^{2}\right) q^{3}}{a^{2}}\left(1-\frac{a b}{q^{4}}\right)
$$

and

$$
Y_{5}=\frac{\left(1+q+q^{2}\right) q}{a}\left(1-\frac{b}{a q}\right)+\frac{q^{6}}{a^{3}}\left(1-\frac{a b}{q^{3}}\right) .
$$

6. Theorem 6, for $k=1$ and 2, produces the results given in [20] (pp. 30-31, Equations (28) and (29)) (Equation (29) with minor corrections). Moreover, for $k=3,4,5, \cdots$, Theorem 6 gives a number of other presumably new $q$-summations. For instance, the case when $k=3$ yields the following result:

$$
\begin{gathered}
{ }_{2} \Phi_{2}\left[\begin{array}{l}
a, \frac{1}{a q^{2}} ; q,-b q^{3} \\
b,-q ;
\end{array}\right]=\frac{a^{3} q^{3}}{\left(1+a q^{2}\right)(1+a q)(1+a)(b ; q)_{\infty}} \\
\cdot\left[\left(\frac{b}{a}, a b q^{3} ; q^{2}\right)_{\infty} X_{6}+\left(\frac{b q}{a}, a b q^{2} ; q^{2}\right)_{\infty} Y_{6}\right],
\end{gathered}
$$

where $X_{6}$ and $Y_{6}$ are as given below:

$$
X_{6}=\left(1-\frac{b}{a q^{2}}\right)+\frac{\left(1+q+q^{2}\right)}{a^{2} q^{3}}\left(1-a b q^{2}\right)
$$

and

$$
Y_{6}=\frac{\left(1+q+q^{2}\right)}{a q^{2}}\left(1-\frac{b}{a q}\right)+\frac{\left(1-a b q^{3}\right)}{a^{3} q^{3}} .
$$

\section{Concluding Remarks and Observations}

In conclusion, this paper establishes a fact that the known techniques, which were used in $[15,16,18]$ to derive the three $q$-Kummer summation theorems, can be applied in a straightforward manner to develop the general contiguous extensions of the $q$-Kummer summation theorems (6), (9) and (10). Moreover, the techniques used in this paper are clearly more efficient and more effective than the use of the contiguous relations by Harsh et al. (see $[19,20])$, because the techniques used here have been demonstrated to be much more widely applicable and in more general situations as well (see also some related earlier works [31-36]) on the subject of our present investigation. In a forthcoming sequel to this article, we aim at investigating several general contiguous extensions of the $q$-analogues of Dixon's summation theorem [7] (p. 355, Equation (II.13)), Watson's summation theorem [7] 
(p. 355, Equation (II.16)) and Whipple's summation theorem [7] (p. 355, Equations (II.18) and (II.19)). This seemingly worthwhile sequence to our present work is under preparation and will be communicated in the foreseeable future.

In view of the applications, which are mentioned in [16] (Sections 2 and 3) regarding derivations of partitions as well as number-theoretic identities from the basic hypergeometric summations and transformations, and also the applications of Watson's transformation in deriving the Rogers-Ramanujan type identities (see [2] (pp. 103-105) and [7] (p. 44)), the results investigated here are potentially useful in these directions.

It may be remarked that, in a recent paper by Srivastava [8], the so-called $(p, q)$ calculus was exposed to be a rather trivial and inconsequential variation of the classical $q$-calculus, since the additional parameter $p$ is redundant (see, for details, Reference [8] (p. 340)). This observation by Srivastava [8] will indeed apply also to any attempt to produce the rather straightforward $(p, q)$-variations of the results which we have presented in this paper.

Author Contributions: Formal analysis, H.M.S.; Investigation, Y.V., H.M.S. and S.P.; Methodology, Y.V. and K.F.; Resources, Y.V., S.P. and K.F.; Software, S.P. and K.F.; Supervision, H.M.S.; Writing-review and editing, H.M.S. All authors have read and agreed to the published version of the manuscript.

Funding: This research received no external funding.

Institutional Review Board Statement: Not applicable.

Informed Consent Statement: Not applicable.

Data Availability Statement: Not applicable.

Conflicts of Interest: The authors declare that they have no conflict of interest.

\section{References}

1. Rainville, E.D. Special Functions; Macmillan Company: New York, NY, USA, 1960; Reprinted by Chelsea Publishing Company: New York, NY, USA, 1971.

2. Slater, L.J. Generalized Hypergeometric Functions; Cambridge University Press: Cambridge, UK, 1966.

3. Srivastava, H.M.; Karlsson, P.W. Multiple Gaussian Hypergeometric Series. In Ellis Horwood Series on Mathematics and Its Applications; John Wiley and Sons: New York, NY, USA, 1985.

4. Srivastava, H.M.; Manocha, H.L. A Treatise on Generating Functions; John Wiley and Sons: New York, NY, USA, 1984.

5. Andrews, G.E.; Askey, R.; Roy, R. Special Functions. In Encyclopedia of Mathematics and Its Applications; Cambridge University Press: Cambridge, UK, 1999; Volume 71.

6. Exton, H. q-Hypergeometric Functions and Applications; John Wiley and Sons: New York, NY, USA, 1983.

7. Gasper, G.; Rahman, M. Basic Hypergeometric Series. In Encyclopedia of Mathematics and Its Applications, 2nd ed.; Cambridge University Press: Cambridge, UK, 2004; Volume 96.

8. Srivastava, H.M. Operators of basic (or $q$-) calculus and fractional $q$-calculus and their applications in geometric function theory of complex analysis. Iran. J. Sci. Technol. Trans. A Sci. 2020, 44, 327-344.

9. Srivastava, H.M.; Cao, J.; Arjika, S. A note on generalized $q$-difference equations and their applications involving $q$-hypergeometric functions. Symmetry 2020, 12, 1816.

10. Kac, V.; Cheung, P. Symmetric quantum calculus. In Quantum Calculus; Universitext Book Series; Springer: Berlin/Heidelberg, Germany, 2002; pp. 99-104.

11. Kummer, E.E. Über die hypergeometrische Reihe $1+\frac{\alpha \cdot \beta}{1 \cdot \gamma} x+\frac{\alpha(\alpha+1) \cdot \beta(\beta+1)}{1 \cdot 2 \cdot \gamma(\gamma+1)} x^{2}+\frac{\alpha(\alpha+1)(\alpha+2) \cdot \beta(\beta+1)(\beta+2)}{1 \cdot 2 \cdot 3 \cdot \gamma(\gamma+1)(\gamma+2)} x^{3}+\cdots$. J. Reine Angew. Math. 1836, 15, 39-83 and 127-172.

12. Choi, J.; Rathie, A.K.; Srivastava, H.M. A generalization of a formula due to Kummer. Integral Transforms Spec. Funct. 2011, 22, 851-859.

13. Daum, J.A. The basic analog of Kummer's theorem. Bull. Amer. Math. Soc. 1942, 48, 711-713.

14. Bailey, W.N. A note on certain $q$-identities. Quart. J. Math. Oxford Ser. 1941, 12, 173-175.

15. Andrews, G.E. On the $q$-analog of Kummer's theorem and applications. Duke Math. J. 1973, 40, 525-528.

16. Andrews, G.E. Applications of basic hypergeometric functions. SIAM Rev. 1974, 16, 441-484.

17. Jackson, F.H. Transformations of $q$-series. Mess. Math. 1910, 39, 145-153.

18. Kim, Y.S.; Rathie, A.K.; Lee, C.H. On $q$-analogue of Kummer's theorem and its contiguous results. Commun. Korean Math. Soc. 2003, 18, 151-157. 
19. Harsh, H.V.; Kim, Y.S.; Rakha, M.A.; Rathie, A.K. A study of $q$-contiguous function relations. Commun. Korean Math. Soc. 2016, 31, 65-94.

20. Harsh, H.V.; Kim, Y.S.; Rathie, A.K.; Purohit, S.D. On basic analogue of classical summation theorems due to Andrews. Honam Math. J. 2016, 38, 25-37.

21. Joshi, C.M.; Vyas, Y. Bailey type transforms and applications. Jñānābha 2015, 45, 53-80.

22. Lin, S.-D.; Srivastava, H.M. Some closed-form evaluations of multiple hypergeometric and $q$-hypergeometric series. Acta Appl. Math. 2005, 86, 309-327.

23. Srivastava, H.M.; Arjika, S. A general family of $q$-hypergeometric polynomials and associated generating functions. Mathematics 2021, 9, 1161.

24. Srivastava, H.M.; Jain, V.K. q-series identities and reducibility of basic double hypergeometric functions. Canad. J. Math. 1986, 38, 215-231.

25. Srivastava, H.M.; Kumar, B.R.S.; Narendra, R. Some modular equations analogous to Ramanujan's identities. Rev. Real Acad. Cienc. Exactas Fís. Natur. Ser. A Mat. 2021, 115, 1-12.

26. Joshi, C.M.; Vyas, Y. Extensions of Bailey's transform and applications. Internat. J. Math. Math. Sci. 2005, 2005, 1909-1923.

27. Agarwal, R.P. The Resonance of Ramanujan's Mathematics; New Age International Publishers: New Delhi, India, 1996; Volumes I-III.

28. Singh, V.N. The basic analogues of identities of Cayley-Orr type. J. Lond. Math. Soc. 1959, 34, 15-22.

29. Choi, J.; Rathie, A.K.; Malani, S. Kummer's theorem and its contiguous identities. Taiwan. J. Math. 2007, 11, 1521-1527.

30. Srivastava, H.M. A note on Jacobi's generating function for the Jacobi polynomials. Proc. Japan Acad. Ser. A Math. Sci. 1985, 61, 201-202.

31. Andrews, G.E. Summations and transformations for basic Appell series. J. Lond. Math. Soc. 1972, 4, 618-622.

32. Maier, R.S. A generalization of Euler's hypergeometric transformation. Trans. Amer. Math. Soc. 2006, 358, 39-57.

33. Maier, R.S. Extensions of the classical transformations of the hypergeometric function ${ }_{3} F_{2}$. Adv. Appl. Math. 2019, 105, 25-47.

34. Srivastava, H.M.; Vyas, Y.; Fatawat, K. Extension of the classical theorems for very well-poised hypergeometric functions. Rev. Real Acad. Cienc. Exactas Fís. Nat. Ser. A Mat. 2019, 113, 367-397.

35. Vidūnas, R. A generalization of Kummer's identity. Rocky Mt. J. Math. 2002, 32, 919-936.

36. Vidūnas, R. Contiguous relations of hypergeometric series. J. Comput. Appl. Math. 2003, 153, 507-519. 